\title{
AUTOMORPHISMS OF BLOWUPS OF THREEFOLDS BEING FANO OR HAVING PICARD NUMBER 1
}

\author{
TUYEN TRUNG TRUONG
}

\begin{abstract}
Let $X_{0}$ be a smooth projective threefold which is Fano or which has Picard number 1. Let $\pi: X \rightarrow X_{0}$ be a finite composition of blowups along smooth centers. We show that for "almost all" of such $X$, if $f \in \operatorname{Aut}(X)$ then its first and second dynamical degrees are the same. We also construct many examples of finite blowups $X \rightarrow X_{0}$, on which any automorphism is of zero entropy.

The main idea is that because of the log-concavity of dynamical systems and the invariance of Chern classes under holomorphic automorphisms, there are some constraints on the nef cohomology classes.

We will also discuss a possible application of these results to a threefold constructed by Kenji Ueno.
\end{abstract}

\section{INTRODUCTION}

While there are many examples of compact complex surfaces having automorphisms of positive entropies (works of Cantat [10, Bedford-Kim [5] [6], McMullen 27] 28] 29] 30, Oguiso 32] 33, Cantat-Dolgachev 11], Zhang [46, Diller 17, Déserti-Grivaux [16, Reschke [38,...), there are few interesting examples of manifolds of higher dimensions having automorphisms of positive entropies (Oguiso 34 35, Oguiso-Perroni 31,...). In particular, for the class of smooth rational threefolds, there are currently only two known examples of manifolds with primitive automorphisms of positive entropy (see [36, 13, 12]). Here a primitive automorphism, defined by D.-Q. Zhang [46], is one that has no non-trivial invariant fibrations over a base of dimension 1 or 2. For general properties on automorphism groups of compact Kähler manifolds, see the recent survey paper [18.

Then, it is natural to ask for what happens in dimension 3 and higher. For example, the following question was asked by Eric Bedford in 2011:

Question 1. Is there a finite composition of blowups at points or smooth curves $X \rightarrow \mathbb{P}^{3}$ starting from $\mathbb{P}^{3}$ and an automorphism $f: X \rightarrow X$ with positive entropy?

This paper aims to study Question 1 and some related questions. We give many evidences to that the answer to Question 1 is negative and to that the examples in [36, 13, 12, can not be obtained as smooth blowups of smooth threefolds having Picard number 1 or being Fano.

Our results and proofs are stated in terms of dynamical degrees, which we recall now. Let $X$ be a smooth projective threefold. We denote by $\operatorname{Pic}(X)$ the Picard group of $X, P i c_{\mathbb{Q}}(X)=\operatorname{Pic}(X) \otimes_{\mathbb{Z}} \mathbb{Q}$ and $P i c_{\mathbb{R}}(X)=\operatorname{Pic}(X) \otimes_{\mathbb{Z}} \mathbb{R}$. Let $N e f(X) \subset$ $P i c_{\mathbb{R}}(X)$ be the cone of nef classes, which is the closure of the cone of ample classes.

Date: July 1, 2018.

2010 Mathematics Subject Classification. 37F, 14D, 32U40, 32H50.

Key words and phrases. Automorphisms, Blowups, Dynamical degrees, Topological entropy. 
By Kleiman's criterion, a class in $P i c_{\mathbb{R}}(X)$ is nef iff it has non-negative intersection with every curve on $X$. For later use, we denote by $c_{1}(X)$ and $c_{2}(X)$ the first and second Chern classes of $X$. Let $f: X \rightarrow X$ be an automorphism. Then $f$ preserves both $\operatorname{Pic}(X)$ and $\operatorname{Nef}(X)$. Let $\omega$ be an ample class on $X$. We define the first and second dynamical degrees of $f$ as follows:

$$
\begin{aligned}
& \lambda_{1}(f)=\lim _{n \rightarrow \infty}\left[\left(f^{n}\right)^{*}(\omega) \cdot \omega^{2}\right]^{1 / n}, \\
& \lambda_{2}(f)=\lim _{n \rightarrow \infty}\left[\left(f^{n}\right)^{*}\left(\omega^{2}\right) \cdot \omega\right]^{1 / n} .
\end{aligned}
$$

Here are some properties of these dynamical degrees: $\lambda_{1}(f)^{2} \geq \lambda_{2}(f) \geq 1$ and $\lambda_{1}\left(f^{-1}\right)=\lambda_{2}(f)$. For more on dynamical degrees see 21 .

Entropy of $f$ can be computed via dynamical degrees by Gromov-Yomdin's theorem 24, 42: $h_{\text {top }}(f)=\log \max \left\{\lambda_{1}(f), \lambda_{2}(f)\right\}$. Hence, $f$ has positive entropy iff $\lambda_{1}(f)>1$.

Primitivity of $f$ can also be detected from dynamical degrees via the following criterion (see [36]), which is a consequence of results in 19] and [20]: If $\lambda_{1}(f) \neq \lambda_{2}(f)$ then $f$ is primitive.

The main idea behind all the results of this paper is that the existence of an automorphism $f$ of positive entropy on $X$ imposes some constraints on the cohomology groups of $X$. In fact, let $0 \neq \zeta \in N e f(X)$ be such that $f^{*}(\zeta)=\lambda_{1}(f) \zeta$ (the existence of such a class is guaranteed by Perron-Frobenius theorem). The differential $d f$ gives an isomorphism between the tangent bundle $T X$ and its pullback $f^{*}(T X)$. Hence, from the properties of Chern classes we have $f^{*} c_{1}(X)=c_{1}(X)$ and $f^{*} c_{2}(X)=c_{2}(X)$. Since $\lambda_{1}(f)>1$ and $X$ has dimension 3 , it follows that

$$
\zeta^{3}=\zeta^{2} \cdot c_{1}(X)=\zeta \cdot c_{1}(X)^{2}=0 .
$$

In fact, stronger constraints are satisfied.

Theorem 1. Let $X$ be a projective manifold of dimension 3 and $f: X \rightarrow X$ an automorphism.

1) If $f$ has positive entropy, there is a nef class $\zeta$ which is not in $\mathbb{R} \cdot$ Pic $_{\mathbb{Q}}(X)$ such that $\zeta^{2}=0, \zeta . c_{1}(X)^{2}=0$ and $\zeta . c_{2}(X)=0$.

2) If $\lambda_{1}(f) \neq \lambda_{2}(f)$, there is a nef class $\zeta$ which is not in $\mathbb{R} . P i c_{\mathbb{Q}}(X)$ such that $\zeta^{2}=0, \zeta . c_{1}(X)=0$ and $\zeta . c_{2}(X)=0$.

Here we comment on the condition $\zeta^{2}=0$. If $X$ has dimension 2 , then this condition is one homogeneous equation in $m$ variables (here, $m$ is the Picard number of $X$ ) and hence is very easily satisfied. In contrast, when $X$ has dimension 3 or bigger, then the condition $\zeta^{2}=0$ is a system of $p \geq m$ homogeneous equations in the $m$ variables (here $p$ is the dimension of $\bigwedge^{2} P i c_{\mathbb{R}}(X)$ ), and hence is more difficult to be satisfied. This is a heuristic argument for why it is difficult to find automorphisms of positive entropy in dimension 3 or larger.

Based on Theorem 1, we state some conditions on nef cohomology classes.

Condition 2. Let $X$ be a smooth projective threefold.

1) Condition A: We say that $X$ satisfies Condition $A$ if whenever $\zeta \in \operatorname{Nef}(X)$ is such that $\zeta^{2}=0, \zeta \cdot c_{1}(X)^{2} \geq 0$ and $\zeta \cdot c_{2}(X) \leq 0$, then $\zeta \in \mathbb{R} . P i c_{\mathbb{Q}}(X)$.

2) Condition B: We say that $X$ satisfies Condition $B$ if whenever $\zeta \in N e f(X)$ is such that $\zeta^{2}=0, \zeta \cdot c_{1}(X)=0$ and $\zeta \cdot c_{2}(X) \leq 0$, then $\zeta \in \mathbb{R} . P i c_{\mathbb{Q}}(X)$. 
By Theorem 1, if $X$ satisfies Condition A then any automorphism on $X$ has zero entropy, and if $X$ satisfies Condition B then for any automorphism $f$ of $X$ we have $\lambda_{1}(f)=\lambda_{2}(f)$. While requiring more than the assumptions in part 1) of Theorem 1. Condition A is very suitable for inductive arguments. A similar comment applies for Condition B.

Now we are ready to state the main results of this paper. The first result is for blowups of some special configurations of $\mathbb{P}^{3}$.

Theorem 3. Let $p_{1}, \ldots, p_{n}$ be distinct points in $X_{0}=\mathbb{P}^{3}$ such that any 4 points of them do not belong to the same hyperplane. Let $C_{i, j}$ be the line connecting the points $p_{i}$ and $p_{j}$. Let $\pi_{1}: X_{1} \rightarrow \mathbb{P}^{3}$ be the blowup at $p_{1}, \ldots, p_{n}$. Let $D_{i, j} \subset X_{1}$ be the strict transforms of $C_{i, j}$, and $\pi_{2}: X_{2} \rightarrow X_{1}$ be the blowup at $D_{i, j}$. Then any automorphism of $X_{2}$ has zero entropy.

Remark. Igor Dolgachev and Yuri Prokhorov informed us that in the special cases where $4 \leq n \leq 7$, then the automorphism group of $X_{2}$ in Theorem 3 is finite. The conclusion of Theorem 3 can be proved for the blowups of more general configurations in $\mathbb{P}^{3}$. However, since the statements of these generalizations are a bit complicated, we refer to Section 4 for more details.

The next two main results of the paper are for threefolds having Picard number 1 or satisfying a special property on the second Chern class. We recall that a class $\zeta$ on $X$ is movable if there is a smooth blowup $\pi: Z \rightarrow X$ such that $\zeta$ is the pushforward of some nef class on $Z$.

Theorem 4. Let $X_{0}$ be a threefold with Picard number 1. Let $C_{1}, \ldots, C_{t} \subset X_{0}$ be smooth curves which are pairwise disjoint. Let $p_{1}, \ldots, p_{s} \in X_{0}$ be distinct points, which are allowed to belong to the curves $C_{1}, \ldots, C_{t}$. Let $\pi_{1}: X_{1} \rightarrow X_{0}$ be the blowup at $p_{1}, \ldots, p_{s}$, and $\pi_{2}: X_{2} \rightarrow X_{1}$ the blowup at $C_{1}, \ldots, C_{t}$. Then $X_{2}$ satisfies Properties $A$ and $B$.

We note that in general Theorem 4 does not hold for threefolds $X_{0}$ with Picard number $\geq 2$ (for example when $\left.X_{0}=\mathbb{P}^{2} \times \mathbb{P}^{1}\right)$. However, the theorems below may still hold for those manifolds. See Section 4 for more details.

Theorem 5. Let $X_{0}$ be a smooth projective threefold such that $c_{2}\left(X_{0}\right) . \zeta>0$ for all non-zero movable $\zeta \in N S_{\mathbb{R}}\left(X_{0}\right)$. Let $p_{1}, \ldots, p_{n} \in X_{0}$ be distinct points. Let $\pi_{1}: X_{1} \rightarrow X_{0}$ be the blowup of $X_{0}$ at $p_{1}, \ldots, p_{n}$. Let $D_{1}, \ldots, D_{m} \subset X_{1}$ be disjoint smooth curves, and $\pi_{2}: X_{2} \rightarrow X_{1}$ the blowup at $D_{1}, \ldots, D_{n}$.

1) $X_{2}$ satisfies Condition $B$.

2) Assume moreover that for any $j$, then $c_{1}\left(X_{1}\right)$. $D_{j} \leq 2 g_{j}-2$, where $g_{j}$ is the genus of $D_{j}$. Then $X_{2}$ satisfies Condition $A$.

Theorem [5 applies for $X_{0}=\mathbb{P}^{3}$ or $\mathbb{P}^{2} \times \mathbb{P}^{1}$ or $\mathbb{P}^{1} \times \mathbb{P}^{1} \times \mathbb{P}^{1}$. It also applies for complete intersection threefolds in $\mathbb{P}^{N}$. See Section 4 for more details. We note that here the images in $X_{0}$ of $D_{1}, \ldots, D_{n}$ may be singular and intersect with each other, hence Theorem 5 is not covered by Theorem 4 even in the case $X_{0}=\mathbb{P}^{3}$.

Finally, we state several results which are purely inductive in nature, which can be applied to blowups of Fano threefolds as well. Here we recall that a threefold is Fano if $c_{1}(X)$ is ample.

We start with the case of point blowups. 
Theorem 6. Let $Y$ be a smooth projective threefold satisfying one of the Conditions $A$ and B. Let $\pi: X \rightarrow Y$ be the blowup at a point. Then $X$ satisfies the same Condition.

Next, we consider the case of curve blowups.

Theorem 7. Let $Y$ be a smooth projective threefold satisfying Condition A or B. Let $\pi: X \rightarrow Y$ be the blowup at a smooth curve $C \subset Y$. Let $g$ be the genus of $C$, and define $\gamma=c_{1}(Y) . C+2 g-2$. Then $X$ also satisfies the same Condition, if one of the following cases happens.

1) $c_{1}(Y) . C$ is an odd number and the normal vector bundle $N_{C / Y}$ is decomposable. The latter means that $N_{C / Y}$ is the direct sum of two line bundles over $C$.

2) $\gamma<0$ and $C$ is not the only effective curve in its cohomology class.

3) There is an irreducible hypersurface $S \subset Y$ such that $2 \kappa<\mu \gamma$. Here $\kappa=S . C$ and $\mu$ is the multiplicity of $C$ in $S$.

We note that in 1) of Theorem[7 the condition that $N_{C / Y}$ is decomposable may be easily to satisfy. For example, if $C$ is a smooth rational curve, then $N_{C / Y}$ is always decomposable by a result of Grothendieck, even if $C$ does not move in $Y$.

Theorem 8. Let $Y$ be a smooth projective threefold satisfying Condition B. Let $\pi: X \rightarrow Y$ be the blowup at a smooth curve $C \subset Y$. Let $g$ be the genus of $C$. If $c_{1}(Y) . C \neq 2 g-2$, then $X$ also satisfies Condition $B$.

Hence, we conclude that if $X_{0}$ is a smooth threefold which is Fano or has Picard number 1 , then for almost every $X \rightarrow X_{0}$ a finite composition of points or smooth curves, every automorphism $f$ on $X$ has $\lambda_{1}(f)=\lambda_{2}(f)$. This is a strong indication that probably all automorphisms on such manifolds are not primitive, i.e. has invariant fibrations over a base of dimension 1 or 2 .

In Section 5 we will discuss possible application of the above results to the Ueno's threefold considered in 41. In Section 4, we will give various examples illustrating the above results.

Remark. The general case of compact Kähler threefolds can be similarly treated, by replacing the Neron-Severi group by the $(1,1)$ cohomology group. After the appearance of a first version of this paper (see [40]), some generalizations to higher dimensions have been given in 2] and 39.

Acknowledgements. The author is grateful to Tien-Cuong Dinh for his suggestion that the answer to Question 1 is negative. The author has been benefited from helpful discussions and correspondences with Ekaterina Amerik, Turgay Bayraktar, Eric Bedford, Frederic Campana, Igor Dolgachev, Mattias Jonsson, Jan-Li Lin Viet-Anh Nguyen, Keiji Oguiso, Yuri Prokhorov, Roland Roeder and Konstantin Shramov.

\section{Preliminaries on NeF Classes AND BlOWUPS}

2.1. Kähler, nef and psef classes, and effective varieties. Let $X$ be a compact Kähler manifold. Let $\eta \in H^{1,1}(X)$. We say that $\eta$ is Kähler if it can be represented by a Kähler $(1,1)$ form. We say that $\eta$ is nef if it is a limit of a sequence of Kähler classes. We say that $\eta$ is psef if it can be represented by a positive closed $(1,1)$

current. A class $\xi \in H^{p, p}(X)$ is an effective variety if there are irreducible varieties 
$C_{1}, \ldots, C_{t}$ of codimension $p$ in $X$ and non-negative real numbers $a_{1}, \ldots, a_{t}$ so that $\xi$ is represented by $\sum_{i} a_{i} C_{i}$.

Demailly and Paun [15] gave a characterization of Kähler and nef classes, which in the case of projective manifolds is summarized as follows:

Theorem 9. Let $X$ be a projective manifold with a Kähler $(1,1)$ form $\omega$. A class $\eta \in H^{1,1}(X)$ is Kähler if and only for any irreducible subvariety $V \subset X$ then $\int_{V} \eta^{\operatorname{dim}(V)}>0$. A class $\eta \in H^{1,1}(X)$ is nef if and only for any irreducible subvariety $V \subset X$ then $\int_{V} \eta^{\operatorname{dim}(V)-j} \wedge \omega^{j} \geq 0$ for all $0 \leq j \leq \operatorname{dim}(V)$.

Nef classes are preserved under pullback by holomorphic maps.

Lemma 1. Let $\pi: X \rightarrow Y$ be a holomorphic map between compact Kähler manifolds. Then $\pi^{*}\left(H_{\text {nef }}^{1,1}(X)\right) \subset H_{\text {nef }}^{1,1}(Y)$.

Proof. Since nef classes are in the closure of Kähler classes, it suffices to show that if $\eta$ is a Kähler class then $\pi^{*}(\eta)$ is nef. Let $\varphi$ be a Kähler $(1,1)$ form representing $\eta$. Then $\pi^{*}(\varphi)$ is a positive smooth $(1,1)$ form. Let $\omega_{X}$ be a Kähler $(1,1)$ form on $X$. Then $\pi^{*}(\eta)$ is represented as a limit of the following Kähler classes

$$
\pi^{*}(\varphi)+\frac{1}{n} \omega_{X}
$$

and hence is nef.

Remark: Similarly, it can be shown that psef classes are preserved under pushforward by holomorphic maps. However, nef classes may not be preserved under pushforwards, even when the map is a blowup.

2.2. Blowup of a projective 3-manifold at a point. Let $\pi: X \rightarrow Y$ be the blowup of a projective 3-manifold at a point $p$. Let $E=\mathbb{P}^{2}$ be the exceptional divisor and let $L \subset E$ be a line. Then $H^{1,1}(X)$ is generated by $\pi^{*}\left(H^{1,1}(Y)\right)$ and $E$, and $H^{2,2}(X)$ is generated by $\pi^{*}\left(H^{2,2}(Y)\right)$ and $L$. The intersection product on the cohomology of $X$ is given by

$$
\begin{gathered}
\pi^{*}(\xi) \cdot E=0, E \cdot E=-L, \\
\pi^{*}(\xi) \cdot L=0, E \cdot L=-1 .
\end{gathered}
$$

The first and second Chern classes of $X$ can be computed by (see e.g. Section 6, Chapter 4 in the book of Griffiths-Harris [25])

$$
\begin{aligned}
& c_{1}(X)=\pi^{*}\left(c_{1}(Y)\right)-2 E, \\
& c_{2}(X)=\pi^{*}\left(c_{2}(Y)\right) .
\end{aligned}
$$

The following result concerns the relations between cycles on $X$ and $Y$.

Lemma 2. For any effective curve $V \subset Y$, there is an effective curve $\widetilde{V} \subset X$ so that $\pi_{*}(\widetilde{V})=V$ and $\widetilde{V} . E \geq 0$.

Proof. It suffices to consider the case when $V$ is an irreducible curve. We can choose $\widetilde{V}$ to be the strict transform of $V$. Then $\pi_{*}(\widetilde{V})=V$, and $\widetilde{V}$ is not contained in $E$. Therefore $\widetilde{V} \cdot E \geq 0$.

We end this subsection showing that nef classes are preserved under pushforward by point-blowups.

Lemma 3. Let $\eta \in H_{n e f}^{1,1}(X)$. Then $\pi_{*}(\eta) \in H_{n e f}^{1,1}(Y)$. 
Proof. It suffices to prove the conclusion when $\eta$ is a Kähler class. Let $\varphi$ be a Kähler $(1,1)$ form representing $\eta$. Then $\pi_{*}(\varphi)$ is a positive closed $(1,1)$ current, which is smooth on $X-p$.

Let $\omega_{Y}$ be a Kähler $(1,1)$ form on $Y$. To show that $\pi_{*}(\eta)$ is a nef class, by Theorem 9 it suffices to show that for any irreducible variety $V \subset Y$ then $\pi_{*}(\eta)^{\operatorname{dim}(V)-j} \cdot V \cdot \omega_{Y}^{j} \geq 0$ for $0 \leq j \leq \operatorname{dim}(V)$. We let $[V]$ be the current of integration on $V$. Then by the results in Section 4, Chapter 3 in the book of Demailly [14, the current $\pi_{*}(\varphi)^{\operatorname{dim}(V)-j} \wedge[V] \wedge \omega_{Y}^{j}$ is well-defined and is a positive measure, whose mass equals to $\pi_{*}(\eta)^{\operatorname{dim}(V)-j} . V \cdot \omega_{Y}^{j}$. Thus the latter quantity is non-negative.

2.3. Blowup of a projective 3-manifold along a smooth curve. Let $\pi: X \rightarrow$ $Y$ be the blowup of a projective 3-manifold along a smooth curve $C \subset Y$. Let $g$ be the genus of $C$. Let $F$ be the exceptional divisor and let $M$ be a fiber of the projection $F \rightarrow C$. We can identify $F$ with the projective bundle $\mathbb{P}(\mathcal{E}) \rightarrow C$, where $\mathcal{E}=N_{C / Y} \rightarrow C$ is the normal vector bundle of $C$ in $Y$.

Then $H^{1,1}(X)$ is generated by $\pi^{*}\left(H^{1,1}(Y)\right)$ and $F$, and $H^{2,2}(X)$ is generated by $\pi^{*}\left(H^{2,2}(Y)\right)$ and $M$. The intersection between $F$ and $M$ is $F . M=-1$. The first and second Chern classes of $X$ can be computed as follows:

$$
\begin{aligned}
& c_{1}(X)=\pi^{*}\left(c_{1}(Y)\right)-F, \\
& c_{2}(X)=\pi^{*}\left(c_{2}(Y)+C\right)-\pi^{*} c_{1}(Y) . F .
\end{aligned}
$$

Let $[F] \rightarrow X$ be the line bundle of $F$ in $X$, and denote by $e=\left.[F]\right|_{F}$. Then (see e.g. Section 6, Chapter 4 in the book of Griffiths - Harris [25]) in $F$ we have the equalities

$$
\text { e. } M=-1 \text {, e.e }=-c_{1}(\mathcal{E}) .
$$

From the SES of vector bundles on $C$

$$
\left.0 \rightarrow T_{C} \rightarrow T_{Y}\right|_{C} \rightarrow \mathcal{E} \rightarrow 0
$$

it follows by the additivity of first Chern classes that

$$
c_{1}(\mathcal{E})=c_{1}\left(T_{Y}\right) \cdot C-c_{1}\left(T_{C}\right)=c_{1}(Y) \cdot C+2 g-2 .
$$

We define

$$
\gamma:=c_{1}(Y) \cdot C+2 g-2 .
$$

Since $F \rightarrow C$ is a ruled surface (i.e. its fibers are projective lines $\mathbb{P}^{1}$ ), there is a canonical section $C_{0}$ which is the image of a holomorphic map $\sigma_{0}: C \rightarrow F$ (see e.g. Section 2, Chapter 5 in Hartshorne's book [26]). Therefore $C_{0}$ is an effective curve in $F$. Such a $C_{0}$ has intersection 1 with a fiber $M$.

We will return to the canonical section $C_{0}$ at the end of this subsection. For now, we however work in a more general assumption on $C_{0}$, for using later. That is, we consider an effective curve $C_{0} \subset F$ with the following properties

$$
\begin{aligned}
C_{0} \cdot C_{0} & =\tau, \\
C_{0} \cdot M & =\mu>0, \\
M \cdot M & =0 .
\end{aligned}
$$

Any divisor on $F$ is numerically equivalent to a linear combination of $C_{0}$ and $M$. We now show the following 
Lemma 4. a)

$$
F . C_{0}=\frac{1}{2}\left(\gamma \mu-\frac{\tau}{\mu}\right)
$$

b)

$$
F . F=-\frac{1}{\mu} C_{0}+\frac{1}{2}\left(\frac{\tau}{\mu^{2}}+\gamma\right) M .
$$

c) $\pi_{*}(F \cdot F)=-C$.

Proof. a) In fact, we have

$$
F . C_{0}=\left.[F]\right|_{C_{0}}=\left.[F]\right|_{F} \cdot C_{0}=e . C_{0},
$$

here the two expressions on the RHS are computed in $F$. On $F$, numerically we can write $e=a C_{0}+b M$. Then from $-1=e \cdot M=\left(a C_{0}+b M\right) \cdot M=a \mu$, we get $a=-1 / \mu$. Substitute this into $e . e=-\gamma$ we obtain

$$
-\gamma=e \cdot e=\left(\frac{1}{\mu} C_{0}-b M\right) \cdot\left(\frac{1}{\mu} C_{0}-b M\right)=\frac{\tau}{\mu^{2}}-2 b,
$$

which implies that

$$
b=\frac{1}{2}\left(\frac{\tau}{\mu^{2}}+\gamma\right)
$$

Therefore

$$
e=\frac{-1}{\mu} C_{0}+\frac{1}{2}\left(\frac{\tau}{\mu^{2}}+\gamma\right) M
$$

Thus

$$
\begin{aligned}
F . C_{0} & =e . C_{0}=\left[\frac{-1}{\mu} C_{0}+\frac{1}{2}\left(\frac{\tau}{\mu^{2}}+\gamma\right) M\right] C_{0} \\
& =\frac{-\tau}{\mu}+\frac{1}{2}\left(\frac{\tau}{\mu}+\gamma \mu\right) \\
& =\frac{1}{2}\left(-\frac{\tau}{\mu}+\gamma \mu\right) .
\end{aligned}
$$

b) From the formula for $e$ in the proof of a) it is not difficult to arrive at the proof of b).

c) Since $C_{0} \cdot M=\mu$, it follows that $\pi_{*}\left(C_{0}\right)=\mu C$. Then from b) we obtain c).

We end this subsection commenting on conditions 2) and 3) of Theorem ??. By Proposition 2.8 in Chapter 5 of [26, there is a line bundle $\mathcal{M} \rightarrow C$ so that the vector bundle $\mathcal{E}^{\prime}=\mathcal{E} \otimes \mathcal{M}$ is normalized in the following sense: $H^{0}\left(\mathcal{E}^{\prime}\right) \neq 0$ but for all line bundle $\mathcal{L} \rightarrow C$ with $c_{1}(\mathcal{L})<0$ then $H^{0}\left(\mathcal{E}^{\prime} \otimes \mathcal{L}\right)=0$. A canonical section $C_{0} \subset F$ can be associated to such a normalized $\mathcal{E}^{\prime}$. The intersection between $C_{0}$ and $M$ is 1 . Moreover, the number

$$
\tau_{0}=C_{0} \cdot C_{0}=c_{1}\left(\mathcal{E}^{\prime}\right)=c_{1}(\mathcal{E})+2 c_{1}(\mathcal{M}),
$$

is an invariant of $F$.

We end this section with some further properties of a ruled surface.

Lemma 5. Assume that the invariant $\tau_{0}$ of $F$ is non-negative. Then

a) For any effective curve $V \subset F$ we have $V . V \geq 0$.

b) If moreover $\gamma<0$ then for any non-zero effective curve $V \subset F$ we have $F . V<0$. 
Proof. a) It suffices to prove for the case $V$ is an irreducible curve. Numerically, we write $V=a C_{0}+b M$. If $V=C_{0}$ then $V . V=\tau_{0} \geq 0$. If $V=M$ then $V . V=0$. Hence we may assume that $V \neq C_{0}, M$.

We consider two cases:

Case 1: $\tau_{0}=0$. By Proposition 2.20 in Chapter 5 in [26, we have $a>0$ and $b \geq 0$. Therefore

$$
V . V=a^{2} \tau_{0}+2 a b \geq 0 .
$$

Case 2: $\tau_{0}>0$. By Proposition 2.21 in Chapter 5 in [26], there are two subcases: Subcase 2.1: $a=1, b \geq 0$. Then

$$
V . V=\tau_{0}+2 b \geq 0 .
$$

Subcase 2.2: $a \geq 2, b \geq-a \tau_{0} / 2$. Then

$$
V . V=a^{2} \tau_{0}+2 a b \geq a^{2} \tau_{0}+2 a\left(-a \tau_{0} / 2\right)=0 .
$$

b) It suffices to prove for the case $V$ is an irreducible curve. If $V=M$ then $F . M=-1<0$. If $V=C_{0}$ then by Lemma 4 with $\tau=\tau_{0} \geq 0$ and $\mu=1$

$$
F . C_{0}=\frac{1}{2}\left(\gamma-\tau_{0}\right) \leq \frac{1}{2} \gamma<0
$$

because $\gamma<0$. Therefore we may assume that $V \neq C_{0}, M$, and then proceed as in the proof of a).

\section{Proofs of the MAin Results}

We make use of the following result (see e.g. [44] and [3]).

Lemma 6. Let $X$ be a smooth projective threefold. Let $f: X \rightarrow X$ be an automorphism. If $\lambda_{1}(f)>1$, then $\lambda_{1}(f)$ is irrational.

For the convenience of the readers, we reproduce the proof of this Lemma here.

Proof. Let $A$ be the matrix of $f^{*}: N S_{\mathbb{R}}(X) \rightarrow N S_{\mathbb{R}}(X)$, then $A$ is an integer matrix, and $\lambda_{1}(f)$ is a real eigenvalue of $A$. Moreover, $A$ is invertible and its inverse $A^{-1}$ is the matrix of the map $\left(f^{-1}\right)^{*}: N S_{\mathbb{R}}(X) \rightarrow N S_{\mathbb{R}}(X)$ hence is also an integer matrix. Therefore $\operatorname{det}(A)= \pm 1$. Thus the characteristic polynomial $P(x)$ of $A$ is a monic polynomial of integer coefficients and $P(0)= \pm 1$. Assume that $\lambda_{1}(f)$ is a rational number. Since $\lambda_{1}(f)$ is an algebraic integer, it follows that $\lambda_{1}(f)$ must be an integer. Then we can write $P(x)=\left(x-\lambda_{1}(f)\right) Q(x)$, here $Q(x)$ is a polynomial of integer coefficients. If $\lambda_{1}(f)>1$ we get a contradiction $\pm 1=P(0)=-\lambda_{1}(f) Q(0)$

Now we give the proofs of the main results.

Proof of Theorem 1, 1) Since $f^{*}$ preserves the cone $N$ ef $(X)$, by a Perron-Frobenius type theorem, there is a non-zero nef class $\eta$ so that $f^{*}(\eta)=\lambda_{1}(f) \eta$. Similarly, there is a non-zero nef class $\eta_{-}$so that $\left(f^{-1}\right)^{*}\left(\eta_{-}\right)=\lambda_{1}\left(f^{-}\right) \eta_{-}$.

Assume that $\lambda_{1}(f)>1$. By the log-concavity of dynamical degrees, we also have $\lambda_{1}\left(f^{-1}\right)>1$. By Lemma 6, both $\lambda_{1}(f)$ and $\lambda_{1}\left(f^{-1}\right)$ are irrational. Hence, both $\zeta$ and $\zeta_{-}$are not in $\mathbb{R} . N S_{\mathbb{Q}}(X)$. It is easy to see that

$$
\begin{aligned}
\zeta . c_{1}(X)^{2} & =\zeta . c_{2}(X)=0 \\
\zeta_{-} . c_{1}(X)^{2} & =\zeta_{-} . c_{2}(X)=0 .
\end{aligned}
$$


To prove 1) it suffices to show that either $\zeta^{2}=0$ or $\zeta_{-}^{2}=0$. Assume otherwise. From $\zeta^{2} \neq 0$ and $f^{*}\left(\zeta^{2}\right)=\lambda_{1}(f)^{2} \zeta^{2}$, we have

$$
\lambda_{1}(f)^{2} \leq \lambda_{2}(f)=\lambda_{1}\left(f^{-1}\right) .
$$

Similarly, from $\zeta_{-}^{2} \neq 0$, we have

$$
\lambda_{1}\left(f^{-1}\right) \geq \lambda_{1}(f)^{2}
$$

Combining these two inequalities, we conclude that $\lambda_{1}(f) \geq \lambda_{1}(f)^{4}$, which contradicts to $\lambda_{1}(f)>1$. This completes the proof of 1$)$.

2) The proof of 2) is similar.

Proof of Theorem 3. 1) For the proof, it suffices to show that for any non-zero nef $\zeta$ on $X=X_{2}$ then either $\zeta . c_{1}(X)^{2} \neq 0$ or $\zeta . c_{2}(X) \neq 0$.

We let $E_{1}, \ldots, E_{n}$ be the exceptional divisors of the blowup $\pi_{1}: X_{1} \rightarrow X_{0}=\mathbb{P}^{3}$. Let $F_{i, j}$ be the exceptional divisors of the blowup $\pi_{2}: X=X_{2} \rightarrow X_{1}$. Then we can write

$$
\begin{aligned}
\zeta & =\pi_{2}^{*}(\xi)-\sum_{i<j} \alpha_{i, j} F_{i, j}, \\
\xi & =\pi_{1}^{*}(u)-\sum_{l} \beta_{l} E_{l} .
\end{aligned}
$$

Here $u$ is nef on $\mathbb{P}^{3}$ and $\alpha_{i, j}, \beta_{l} \geq 0$.

For the proof of 1$)$, it then suffices to show that $\operatorname{deg}(u)=0$. From

$$
c_{2}(X)=\pi_{2}^{*} c_{2}\left(X_{1}\right)+\sum_{i<j} \pi_{2}^{*} D_{i, j}-\sum_{i<j} \pi_{2}^{*} c_{1}\left(X_{1}\right) \cdot F_{i, j}
$$

and the fact that $c_{1}\left(X_{1}\right) \cdot D_{i, j}=0$, the condition $\zeta \cdot c_{2}(X)=0$ becomes $\xi \cdot c_{2}\left(X_{1}\right)+$ $\sum_{i<j} \xi \cdot D_{i, j}=0$. Since $c_{2}\left(X_{1}\right)=\pi_{1}^{*}\left(c_{2}\left(\mathbb{P}^{3}\right)\right)$, it follows that $\xi \cdot c_{2}\left(X_{1}\right)=16 \operatorname{deg}(u)$. We also have that $\xi . D_{i, j}=\operatorname{deg}(u)-\beta_{i}-\beta_{j}$ for every $i<j$. Therefore, we obtain

$$
\begin{aligned}
6 \operatorname{deg}(u) & =-\sum_{i<j} \xi \cdot D_{i, j}, \\
\left(6+\frac{n(n-1)}{2}\right) \operatorname{deg}(u) & =(n-1) \sum_{l} \beta_{l} .
\end{aligned}
$$

From the condition $\zeta \cdot c_{1}(X)^{2}=0$, we obtain

$$
\begin{aligned}
0 & =\zeta \cdot c_{1}(X)^{2}=\left(\pi_{2}^{*}(\xi)-\sum_{i<j} \alpha_{i, j} F_{i, j}\right) \cdot\left(\pi_{2}^{*} c_{1}\left(X_{1}\right)^{2}-2 \sum_{i<j} \pi_{2}^{*} c_{1}\left(X_{1}\right) \cdot F_{i, j}+\sum_{i<j} F_{i, j}^{2}\right) \\
& =\xi \cdot c_{1}\left(X_{1}\right)^{2}-\sum_{i<j} \xi \cdot D_{i, j}-2 \sum_{i<j} \alpha_{i, j} c_{1}\left(X_{1}\right) \cdot D_{i, j}+\sum_{i<j} \alpha_{i, j}\left(c_{1}\left(X_{1}\right) \cdot D_{i, j}+2 g_{i, j}-2\right) \\
& =22 \operatorname{deg}(u)-4 \sum_{l} \beta_{l}+\sum_{i<j} \alpha_{i, j}\left(2 g_{i, j}-2-c_{1}\left(X_{1}\right) \cdot D_{i, j}\right) \\
& =22 \operatorname{deg}(u)-4 \sum_{l} \beta_{l}-2 \sum_{i<j} \alpha_{i, j} .
\end{aligned}
$$

In the above, $g_{i, j}=0$ is the genus of $C_{i, j}$, and $c_{1}\left(X_{1}\right) . D_{i, j}=0$ for all $i<j$. In particular, we obtain

$$
\frac{11}{2} \operatorname{deg}(u) \geq \sum_{l} \beta_{l}=\left(\frac{6}{n-1}+\frac{n}{2}\right) \operatorname{deg}(u)
$$


From the above inequality, we will finish showing that $\operatorname{deg}(u)=0$. We consider several cases:

Case 1: $n \geq 10$. From Equation (3.1), it follows immediately that $\operatorname{deg}(u)=0$ as wanted.

Case 2: $6 \leq n \leq 9$. In this case, for each 6 points $p_{i_{1}}, \ldots, p_{i_{6}}$ among $n$ points $p_{1}, \ldots, p_{n}$, there is a unique rational normal curve $C \subset \mathbb{P}^{3}$ of degree 3 passing through the 6 chosen points. Let $D \subset X_{1}$ be the strict transform of $C$. Then $D$ is different from the curves $D_{i, j}$. Therefore $\pi_{2}^{*} D$ is an effective curve, and hence

$$
3 \operatorname{deg}(u)-\sum_{l=1}^{6} \beta_{i_{l}} \geq \xi . D=\zeta . \pi_{2}^{*}(D) \geq 0 .
$$

Summing over all such choices of $p_{i_{1}}, \ldots, p_{i_{n}}$ we find that

$$
\frac{n}{2} \operatorname{deg}(u) \geq \sum_{l} \beta_{l} \text {. }
$$

Combining this with

$$
\sum_{l} \beta_{l}=\left(\frac{6}{n-1}+\frac{n}{2}\right) \operatorname{deg}(u),
$$

we obtain $\operatorname{deg}(u)=0$.

Case 3: $n=4,5$. In this case, we use rational normal curves to obtain

$$
\frac{n}{3} \operatorname{deg}(u) \geq \sum_{l} \beta_{l} \text {. }
$$

Combining this with

$$
\sum_{l} \beta_{l}=\left(\frac{6}{n-1}+\frac{n}{2}\right) \operatorname{deg}(u)
$$

we obtain $\operatorname{deg}(u)=0$.

Case 4: $n=1,2,3$. In this case we have $n \operatorname{deg}(u) \geq \sum_{l} \beta_{l}$. Combining this with

$$
\sum_{l} \beta_{l}=\left(\frac{6}{n-1}+\frac{n}{2}\right) \operatorname{deg}(u)
$$

we obtain $\operatorname{deg}(u)=0$.

Proof. (Proof of Theorem 4)

Let $\pi_{1}^{\prime}: X_{1}^{\prime} \rightarrow X_{0}$ be the blowup at the $C_{1}, \ldots, C_{t}$. Let $F_{1}, \ldots, F_{t}$ be the exceptional divisors. Let $M_{j}=\left(\pi_{1}^{\prime}\right)^{-1}\left(p_{j}\right)$ be the preimages of the points $p_{j}(j=$ $1, \ldots, n)$. These are smooth rational curves, and are among the fibers of the maps $F_{1} \rightarrow C_{1}, \ldots, F_{t} \rightarrow C_{t}$. Let $\pi_{2}^{\prime}: X_{2}^{\prime} \rightarrow X_{1}^{\prime}$ be the blowup at the curves $M_{j}$. Then $X_{2}$ is isomorphic to $X_{2}^{\prime}$.

Fixed a number $j$. Let $i$ be such that $M_{j} \subset F_{i}$. Using that

$$
c_{1}\left(N_{M_{j} / X_{1}^{\prime}}\right)=c_{1}\left(N_{M_{j} / F_{i}}\right)+c_{1}\left(\left.N_{F_{i} / X_{1}}\right|_{M_{j}}\right)=0+(-1)=-1,
$$

we find that

$$
c_{1}\left(X_{1}^{\prime}\right) \cdot M_{j}=1
$$

is an odd number. Therefore, using either part 1) or part 3) of Theorem 7 for the proof of Theorem 5 it suffices to show that $X_{1}^{\prime}$ satisfies both Conditions A and B. To this end, we only need to show that if $\zeta \in N e f\left(X_{1}^{\prime}\right)$ is such that $\zeta^{2}=0$ then $\zeta \in \mathbb{R} \cdot N S_{\mathbb{Q}}\left(X_{1}^{\prime}\right)$. 
Let $H \in N S_{\mathbb{Q}}\left(X_{0}\right)$ be an ample divisor. Since $X_{0}$ has Picard number 1, we can write

$$
\zeta=a\left(\pi_{1}^{\prime}\right)^{*}(H)-\sum_{j} \alpha_{j} F_{j}
$$

where $a, \alpha_{1}, \ldots, \alpha_{t} \geq 0$. If $a=0$, then from the fact that $\zeta$ is nef, we have $\alpha_{1}=\ldots=\alpha_{t}=0$. Therefore, we may assume that $\alpha>0$, and after dividing by $\alpha$ we may assume that $\alpha=1$. Then, for the proof of the theorem, it suffices to show that all the numbers $\alpha_{1}, \ldots, \alpha_{t}$ are in $\mathbb{Q}$.

Since the curves $C_{j}$ are pairwise disjoint, for any $i=1, \ldots, t$ we have

$$
\begin{aligned}
0 & =\zeta^{2} \cdot F_{i}=\left(\left(\pi_{1}^{\prime}\right)^{*} H-\sum_{j} \alpha_{j} F_{j}\right)^{2} \cdot F_{i} \\
& =\left(\pi_{1}^{\prime}\right)^{*} H^{2} \cdot F_{i}-2 \alpha_{i}\left(\pi_{1}^{\prime}\right)^{*} H \cdot F_{i}^{2}+\alpha_{i}^{2} F_{i}^{3} \\
& =2 \alpha_{i} H \cdot C_{i}-\alpha_{i}^{2}\left(c_{1}\left(X_{0}\right) \cdot C_{i}+2 g_{i}-2\right),
\end{aligned}
$$

here $g_{i}$ is the genus of $C_{i}$. We note that $H . C_{i}$ is a positive rational number. Hence, either $\alpha_{i}=0$, or

$$
\alpha_{i}=2 H \cdot C_{i} /\left(c_{1}\left(X_{0}\right) \cdot C_{i}+2 g_{i}-2\right) .
$$

In both cases, $\alpha_{i}$ are rational numbers as wanted.

Proof of Theorem 5. 1) Let $\zeta$ be a nef class on $X_{2}$ such that $\zeta^{2}=0, \zeta . c_{1}(X)=0$ and $\zeta \cdot c_{1}\left(X_{2}\right)^{2} \leq 0$. We need to show that $\zeta \in \mathbb{R} . H_{\text {alg }}^{2}\left(X_{2}, \mathbb{Q}\right)$. More strongly, we will show that $\zeta$ must be 0 .

Let us denote by $F_{j}$ the exceptional divisor over $D_{j}$ of the blowup $\pi_{2}: X_{2} \rightarrow X_{1}$. We denote by $\pi_{1}: X_{1} \rightarrow X_{0}$ the blowup of $C_{0}$ at the points $p_{i}$.

We can write $\zeta=\pi_{2}^{*}(\xi)-\sum_{j} \alpha_{j} F_{j}$, where $\alpha_{j} \geq 0$ and $\xi$ is a movable class on $X_{1}$. Since $D_{j}$ are disjoint, by intersecting the equations $\zeta^{2}=\zeta . c_{1}\left(X_{2}\right)=0$ with $F_{j}$, we find as in [40] that either $\alpha_{j}=0$ or

$$
\xi . D_{j}=\alpha_{j} c_{1}\left(X_{1}\right) \cdot D_{j}=\alpha_{j}\left(2 g_{j}-2\right) .
$$

If $\alpha_{j}=0$ then

$$
\xi . D_{j}=\zeta . D_{j}^{\prime} \geq 0=\alpha_{j} c_{1}\left(X_{1}\right) \cdot D_{j}
$$

where $D_{j}^{\prime} \subset F_{j}$ is a section whose pushforward is $D_{j}$. If $\alpha_{j} \neq 0$ then $\xi . D_{j}=$ $c_{1}\left(X_{1}\right) \cdot D_{j}$. Therefore,

$$
\begin{aligned}
0 \geq \zeta \cdot c_{2}\left(X_{2}\right) & =\left(\pi_{2}^{*}(\xi)-\sum_{j} \alpha_{j} F_{j}\right) \cdot\left(\pi_{2}^{*} c_{2}\left(X_{1}\right)+\sum_{j}\left(\pi_{2}^{*} D_{j}-\pi_{2}^{*} c_{1}\left(X_{j}\right) \cdot F_{j}\right)\right) \\
& =\xi \cdot c_{2}\left(X_{1}\right)+\sum_{j}\left(\xi \cdot D_{j}-\alpha_{j} c_{1}\left(X_{1}\right) \cdot D_{j}\right) .
\end{aligned}
$$

Since each term $\xi \cdot D_{j}-\alpha_{j} c_{1}\left(X_{1}\right) . D_{j}$ is non-negative, we find that $\xi \cdot c_{2}\left(X_{1}\right) \leq 0$. Because $c_{2}\left(X_{1}\right)=\pi_{1}^{*} c_{2}\left(X_{0}\right)$, we then get that $\left(\pi_{1}\right)_{*}(\xi) \cdot c_{2}\left(X_{0}\right) \leq 0$. Because $\left(\pi_{1}\right)_{*}(\xi)$ is movable in $X_{0}$, from the assumption on $c_{2}\left(X_{0}\right)$ we obtain $\left(\pi_{1}\right)_{*}(\xi)=0$. From this, it easy follows that $\xi$ and then $\zeta$ are 0 .

2) The proof is similar to that of 1 ). The difference is now that here for each $j$, either $\alpha_{j}=0$ or

$$
\xi . D_{j}-\alpha_{j} c_{1}\left(X_{1}\right) \cdot D_{j}=\frac{\alpha_{j}}{2}\left[\left(2 g_{j}-2\right)-c_{1}\left(X_{1}\right) \cdot D_{j}\right] .
$$


In the first case

$$
\xi . D_{j}-\alpha_{j} c_{1}\left(X_{1}\right) \cdot D_{j}=\xi \cdot D_{j}=\zeta \cdot D_{j}^{\prime} \geq 0,
$$

where $D_{j}^{\prime} \subset F_{j}$ is a section. In the second case, by the assumption $\left(2 g_{j}-2\right)-$ $c_{1}\left(X_{1}\right) \cdot D_{j} \geq 0$, we also have $\xi \cdot D_{j}-\alpha_{j} c_{1}\left(X_{1}\right) \cdot D_{j} \geq 0$.

Hence,

$$
0 \geq-\sum_{j}\left(\xi \cdot D_{j}-\alpha_{j} c_{1}\left(X_{1}\right) \cdot D_{j}\right) \geq \xi \cdot c_{2}\left(X_{1}\right) .
$$

Then we can proceed as before.

Proof of Theorem [6. Let $F$ be the exceptional divisor of the blowup $\pi$. Let $\zeta$ be a nef class on $X$. Then we can write $\zeta=\pi^{*}(\xi)-\alpha F$ for some $\alpha \geq 0$ and for some movable class $\xi=\pi_{*}(\zeta)$ on $Y$.

Assume that $\zeta^{2}=0$. Then,

$$
0=\zeta^{2}=\left(\pi^{*}(\xi)-\alpha F\right)^{2}=\pi^{*}\left(\xi^{2}\right)+\alpha^{2} F^{2} .
$$

Here we used that $\pi^{*}(\xi) \cdot F=0$. Because the classes of $\pi^{*}\left(\xi^{2}\right)$ and $F^{2}$ are linearly independent in the $(2,2)$ cohomology group of $X$, from the above we have that $\alpha=0$. Then it follows that $\xi$ is nef on $Y$, and $\xi^{2}=0$. Moreover, since $c_{1}(X)=$ $\pi^{*} c_{1}(Y)-2 F$ and $c_{2}(X)=\pi^{*} c_{2}(Y)$ (see Chapter 4 in [25]), we have

$$
\begin{aligned}
\xi \cdot c_{1}(Y) & =\pi_{*}\left(\pi^{*}(\xi) \cdot \pi^{*}\left(c_{1}(Y)\right)\right)=\pi_{*}\left(\pi^{*}(\xi) \cdot\left(\pi^{*} c_{1}(Y)-2 F\right)\right)=\pi_{*}\left(\zeta \cdot c_{1}(X)\right) \\
\xi . c_{1}(Y)^{2} & =\zeta \cdot c_{1}(X)^{2} \\
\xi . c_{2}(Y) & =\zeta \cdot c_{2}(X) .
\end{aligned}
$$

Then, it follows easily that if $Y$ satisfies one of the Conditions A and $\mathrm{B}$, then $X$ also satisfies the same Condition.

Proof of Theorem 7. We will show that if $Y$ satisfies Condition A then $X$ also satisfies Condition A. The proof for Condition B is similar.

Let $\zeta$ be a nef class on $X$. We need to show that if

$$
\begin{aligned}
\zeta^{2} & =0 \\
\zeta . c_{1}(X)^{2} & \geq 0 \\
\zeta . c_{2}(X) & \leq 0
\end{aligned}
$$

then $\zeta \in \mathbb{R} . N S_{\mathbb{Q}}(X)$.

Let $F$ be the exceptional divisor of the blowup $\pi: X \rightarrow Y$. We can write $\zeta=\pi^{*}(\xi)-\alpha F$ for some $\alpha \geq 0$. We also have (see Section 2)

$$
\begin{aligned}
c_{1}(X) & =\pi^{*} c_{1}(Y)-F \\
c_{2}(X) & =\pi^{*} c_{2}(Y)+\pi^{*} C-\pi^{*} c_{1}(Y) \cdot F, \\
\pi_{*}(F . F) & =-C .
\end{aligned}
$$


We first consider the case $\alpha=0$. Then, $\xi$ is nef on $Y$ and moreover $\xi^{2}=0$. We have in this case

$$
\begin{aligned}
\pi_{*}\left(\zeta \cdot c_{1}(X)\right) & =\pi_{*}\left(\pi^{*}(\xi) \cdot\left(\pi^{*} c_{1}(Y)-F\right)\right)=\xi \cdot c_{1}(Y), \\
\zeta \cdot c_{1}(X)^{2} & =\pi^{*}(\xi) \cdot\left(\pi^{*} c_{1}(Y)-F\right)^{2}=\pi^{*}(\xi) \cdot\left(\pi^{*} c_{1}(Y)^{2}-2 \pi^{*} c_{1}(Y) \cdot F+F^{2}\right) \\
& =\xi \cdot c_{1}(Y)^{2}-\xi \cdot C \\
\zeta \cdot c_{2}(X) & =\pi^{*}(\xi) \cdot\left(\pi^{*} c_{2}(Y)+\pi^{*} C-\pi^{*} c_{1}(Y) \cdot F\right) \\
& =\xi \cdot c_{2}(Y)+\xi \cdot C .
\end{aligned}
$$

Since $\xi$ is nef and $C$ is an effective curve, we have $\xi . C \geq 0$. Therefore, from the assumptions $\zeta . c_{1}(X)^{2} \geq 0$ and $\zeta . c_{2}(Y) \leq 0$ we obtain

$$
\begin{aligned}
\xi^{2} & =0, \\
\xi . c_{1}(Y)^{2} & =\zeta \cdot c_{1}(X)^{2}+\xi . C \geq 0, \\
\xi . c_{2}(Y) & =\zeta . c_{2}(X)-\xi . C \leq 0 .
\end{aligned}
$$

Since $Y$ satisfies Condition A by assumption, it follows that $\xi \in \mathbb{R} . N S_{\mathbb{Q}}(Y)$. Then $\zeta=\pi^{*}(\xi) \in \mathbb{R} . N S_{\mathbb{Q}}(X)$. Hence, $X$ also satisfies Condition A.

Now we show that under the assumptions of Theorem [6 then actually $\alpha$ must be 0 . Assume otherwise, i.e. that $\alpha>0$, we will obtain a contradiction. We recall that $\gamma=c_{1}(Y) . C+2 g-2$. From the assumption that $\zeta^{2}=0$ we have

$$
\begin{aligned}
0 & =\zeta^{2} \cdot F=\left(\pi^{*}(\xi)-\alpha F\right)^{2} \cdot F \\
& =\pi^{*}\left(\xi^{2}\right) \cdot F-2 \alpha \pi^{*}(\xi) \cdot F^{2}+F^{3} \\
& =\alpha \xi \cdot C-2 \alpha^{2} \cdot \gamma .
\end{aligned}
$$

In the fourth equality we used the results in Section 2 The assumption that $\alpha>0$ implies that

$$
\xi . C=\alpha \cdot \gamma / 2 .
$$

We now proceed corresponding to parts 1), 2) and 3) of the theorem.

1) In this case $c_{1}(Y) . C$ is an odd number and $N_{C / Y}$ is decomposable. We have a SES of vector bundles over $C$ :

$$
\left.0 \rightarrow T_{C} \rightarrow T_{Y}\right|_{C} \rightarrow N_{C / Y} \rightarrow 0
$$

From this, it follows that

$$
c_{1}\left(N_{C / Y}\right)=c_{1}(Y) \cdot C+2 g-2=\gamma .
$$

Recall that $F$ is the exceptional divisor of the blowup $\pi$. Then $F=\mathbb{P}\left(N_{C / X}\right) \rightarrow$ $C$ is a ruled surface over $C$. Hence, (see Proposition 2.8 in Chapter 5 in [26]) there is a line bundle $\mathcal{M}$ over $C$ such that $\mathcal{E}=N_{C / Y} \otimes \mathcal{M}$ is normalized, in the sense that $H^{0}(\mathcal{E}) \neq 0$, but for every line bundle $\mathcal{L}$ with $c_{1}(\mathcal{L})<0$ then $H^{0}(\mathcal{E} \otimes \mathcal{L})=0$.

Let $f$ be a fiber of the fibration $F \rightarrow C$. Then, (see Proposition 2.9 in Chapter 5 in [26]), there is a so-called zero section $C_{0} \subset F$ with the following properties:

$$
\begin{aligned}
\tau:=C_{0} \cdot C_{0} & =c_{1}(\mathcal{E}), \\
C_{0} \cdot f & =1 .
\end{aligned}
$$

Because $N_{C / Y}$ is decomposable, $\mathcal{E}$ is also decomposable. By part a) of Theorem 2.12 in Section 5 in [26], $c_{1}(\mathcal{E}) \leq 0$. Moreover, from

$$
c_{1}(\mathcal{E})=c_{1}\left(\mathcal{N}_{\mathcal{C} / \mathcal{Y}}\right)+2 c_{1}(\mathcal{M})=c_{1}(Y) \cdot C+2 g-2+2 c_{1}(\mathcal{M}),
$$


and the assumption that $c_{1}(Y) . C$ is an odd number, we get that $c_{1}(\mathcal{E})<0$. Hence $\tau<0$.

From the results in Section 2 we have

$$
C_{0}=-F \cdot F+\frac{1}{2}(\tau+\gamma) f .
$$

Now we obtain the desired contradiction. Since $\zeta$ is nef and $C_{0}$ is an effective curve, we have $\zeta . C_{0} \geq 0$. Hence,

$$
\begin{aligned}
0 & \leq\left(\pi^{*}(\xi)-\alpha F\right) \cdot\left(-F \cdot F+\frac{1}{2}(\tau+\gamma) f\right. \\
& =\xi \cdot \pi_{*}(-F \cdot F)+\alpha F \cdot F \cdot F-\frac{1}{2} \alpha(\tau+\gamma) F . f \\
& =\xi \cdot C-\alpha \gamma+\frac{1}{2} \alpha(\tau+\gamma)=\frac{\alpha \tau}{2}<0 .
\end{aligned}
$$

In the above we used that $\pi_{*}(-F . F)=\pi_{*}\left(C_{0}\right)=C$ (see for example Lemma 4 in (40]), F.f $=-1, F . F . F=-\gamma, \xi . C=\alpha \gamma / 2, \alpha>0$ and $\tau=C_{0} . C_{0}<0$.

2) In this case, $\gamma<0$ and $C$ is not the only effective curve in its cohomology class. Let $D$ be another curve in the cohomology class of $C$. Since $C$ is irreducible, we can assume that $C$ is not contained in the support of $D$. Then $\pi^{*}(D)$ is an effective curve in $X$. Since $\zeta$ is nef, we obtain a contradiction

$$
0 \leq \pi^{*}(D) \cdot \zeta=D \cdot \pi_{*}(\zeta)=D \cdot \xi=C \cdot \xi=\alpha \gamma / 2<0 .
$$

3) In this case, there is an irreducible hypersurface $S \subset Y$ such that $2 \kappa<\mu \gamma$. Here $\kappa=S . C$ and $\mu$ is the multiplicity of $C$ in $S$. We now construct an effective curve $C_{0} \subset F$ and use it to derive a contradiction.

The strict transform $\widetilde{S}$ of $S$ is given by $\widetilde{S}=\pi^{*}(S)-\mu F$, and is an irreducible hypersurface of $X$. Since $\widetilde{S}$ and $F$ are different irreducible hypersurfaces, their intersection $C_{0}=\widetilde{S} \cdot F=\left(\pi^{*}(S)-\mu F\right) \cdot F$ is an effective curve of $F$. We now compute the numbers $C_{0} . C_{0}$ and $C_{0} . M$. We have

$$
\begin{aligned}
C_{0} \cdot C_{0} & =\left.\left.\widetilde{S}\right|_{F} \cdot \widetilde{S}\right|_{F}=\widetilde{S} \cdot \widetilde{S} \cdot F \\
& =\left(\pi^{*}(S)-\mu F\right) \cdot\left(\pi^{*}(S)-\mu F\right) \cdot F=-2 \mu \pi^{*}(S) \cdot F \cdot F+\mu^{2} F \cdot F \cdot F \\
& =2 \mu S \cdot C-\mu^{2} \gamma=2 \mu \kappa-\mu^{2} \gamma .
\end{aligned}
$$

Denote by $\tau=C_{0} \cdot C_{0}$ and $\mu_{0}=C_{0} \cdot M$. Note that $\mu_{0} \neq 0$, otherwise we have $C_{0}$ is a multiplicity of $M$, and hence $\pi_{*}\left(C_{0}\right)=0$. But from the definition of $C_{0}$ we can see that $\pi_{*}\left(C_{0}\right)=\mu C \neq 0$. Then by the computations in Section 2] we have

$$
F . F=-\frac{1}{\mu_{0}} C_{0}+\frac{1}{2}\left(\frac{\tau}{\mu_{0}^{2}}+\gamma\right) M .
$$

Pushforward this by the map $\pi$, using that $\pi_{*}(F . F)=-C$ and $\pi_{*}\left(C_{0}\right)=\mu C$ we have that $\mu_{0}=\mu$.

From the above computation $\tau=2 \mu \kappa-\mu^{2} \gamma$, we obtain

$$
F . C_{0}=\frac{1}{2}\left(\gamma \mu-\frac{\tau}{\mu}\right)=\gamma \mu-\kappa .
$$


Because $\zeta$ is nef, it follows that

$$
\begin{aligned}
0 & \leq \zeta \cdot C_{0}=\left(\pi^{*}(\xi)-\alpha F\right) \cdot C_{0}=\mu \xi \cdot C-\frac{\alpha}{2}\left(\gamma \mu-\frac{\tau}{\mu}\right), \\
& =\frac{\alpha}{2} \gamma \mu-\frac{\alpha}{2}\left(\gamma \mu-\frac{\tau}{\mu}\right)=\frac{\alpha}{2} \frac{\tau}{\mu}=\alpha\left(\kappa-\frac{1}{2} \gamma \mu\right) .
\end{aligned}
$$

This contradicts the assumptions that $2 \kappa<\gamma \mu$ and $\alpha>0$.

Proof of Theorem 8. Let $\zeta$ be a nef class on $X$. We need to show that if

$$
\begin{aligned}
\zeta^{2} & =0 \\
\zeta . c_{1}(X) & =0 \\
\zeta . c_{2}(X) & \leq 0
\end{aligned}
$$

then $\zeta \in \mathbb{R} . N S_{\mathbb{Q}}(X)$.

Let $F$ be the exceptional divisor of the blowup $\pi: X \rightarrow Y$. We can write $\zeta=\pi^{*}(\xi)-\alpha F$ for some $\alpha \geq 0$. As in the proof of Theorem 7 , it suffices to show that $\alpha=0$. We assume otherwise that $\alpha>0$. Let $f \subset F$ be a fiber of the projection $F \rightarrow C$. We have

$$
\begin{aligned}
0 & =\zeta \cdot \zeta=\left(\pi^{*}(\xi)-\alpha F\right) \cdot\left(\pi^{*}(\xi)-\alpha F\right) \\
& =\pi^{*}(\xi \cdot \xi)-2 \alpha \pi^{*}(\xi) \cdot F+\alpha^{2} F \cdot F \\
0 & =\zeta \cdot c_{1}(X)=\left(p^{*}(\xi)-\alpha F\right) \cdot\left(\pi^{*} c_{1}(Y)-F\right) \\
& =\pi^{*}\left(\xi \cdot c_{1}(Y)\right)-\pi^{*}(\xi) \cdot F-\pi^{*} c_{1}(Y) \cdot F+\alpha F^{2} .
\end{aligned}
$$

Intersecting both of these equations with $F$, using $F . F . F=-\gamma$ and $\pi_{*}(F . F)=-C$, we obtain

$$
\begin{aligned}
& 2 \alpha \xi . C-\alpha^{2} \gamma=0, \\
& \alpha c_{1}(Y) . C+\xi . C-\alpha \gamma=0 .
\end{aligned}
$$

Then we must have $\alpha=0$. Otherwise, dividing $2 \alpha$ from the first equation we have that $\xi . C=\alpha \gamma / 2$. Substituting this into the second equation and dividing by $\alpha$ we get $2 c_{1}(Y) \cdot C=\gamma$. Hence $c_{1}(Y) \cdot C=2 g-2$, which is a contradiction.

\section{EXAMPLeS}

4.1. The case $X_{0}=\mathbb{P}^{2} \times \mathbb{P}^{1}$. The Picard number of $X_{0}$ is 2. By Künneth's formula, $H^{1,1}\left(X_{0}\right)$ is generated by the classes of $\mathbb{P}^{2} \times\{p t\}$ and $\mathbb{P}^{1} \times \mathbb{P}^{1}$ (here $\{p t\}$ means a point). The intersection on $H^{1,1}\left(X_{0}\right)$ is

$$
\begin{aligned}
\mathbb{P}^{2} \times\{p t\} . \mathbb{P}^{2} \times\{p t\} & =0 \\
\mathbb{P}^{2} \times\{p t\} . \mathbb{P}^{1} \times \mathbb{P}^{1} & =\mathbb{P}^{1} \times\{p t\} \\
\mathbb{P}^{1} \times \mathbb{P}^{1} . \mathbb{P}^{1} \times \mathbb{P}^{1} & =\{p t\} \times \mathbb{P}^{1}
\end{aligned}
$$

By Künneth's formula again, $H^{2,2}\left(X_{0}\right)$ is generated by $\mathbb{P}^{1} \times\{p t\}$ and $\{p t\} \times \mathbb{P}^{1}$. The pairing between $H^{1,1}\left(X_{0}\right)$ and $H^{2,2}\left(X_{0}\right)$ is given by

$$
\begin{aligned}
\mathbb{P}^{2} \times\{p t\} \cdot \mathbb{P}^{1} \times\{p t\} & =0 \\
\mathbb{P}^{2} \times\{p t\} \cdot\{p t\} \times \mathbb{P}^{1} & =1 \\
\mathbb{P}^{1} \times \mathbb{P}^{1} \cdot \mathbb{P}^{1} \times\{p t\} & =1 \\
\mathbb{P}^{1} \times \mathbb{P}^{1} \cdot\{p t\} \times \mathbb{P}^{1} & =0
\end{aligned}
$$


By Whitney's formula, we have

$$
\begin{aligned}
& c_{1}\left(X_{0}\right)=2 \mathbb{P}^{2} \times\{p t\}+3 \mathbb{P}^{1} \times \mathbb{P}^{1}, \\
& c_{2}\left(X_{0}\right)=6 \mathbb{P}^{1} \times\{p t\}+3\{p t\} \times \mathbb{P}^{1} .
\end{aligned}
$$

Therefore, we can check that $X_{0}$ satisfies all the conditions of Theorems 5,6 , 7 and 8 . In particular, if $D_{1}, \ldots, D_{n} \subset X_{0}$ are pairwise disjoint smooth curves, and $\pi_{1}: X_{1} \rightarrow X_{0}$ is the blowup at $D_{1}, \ldots, D_{n}$, then for any automorphism $f$ of $X_{1}$ we have $\lambda_{1}(f)=\lambda_{2}(f)$. However, $X_{0}$ does not satisfy the conditions of Theorem 4. its Picard number is $2>1$. For an appropriate choice of curves $D_{1}, \ldots, D_{n}$, the threefold $X_{1}$ has automorphisms of positive entropy. In fact, there is a rational surface $S$ obtained from $\mathbb{P}^{2}$ by blowing up distinct points $p_{1}, \ldots, p_{n} \in \mathbb{P}^{2}$ such that $S$ has an automorphism of positive entropy. If we choose $D_{j}=p_{j} \times \mathbb{P}^{1}$, then $D_{j}$ are smooth rational curves which are disjoint, and $X_{1}$ has an automorphism of positive entropy.

4.2. The case $X_{0}=\mathbb{P}^{1} \times \mathbb{P}^{1} \times \mathbb{P}^{1}$. This case is very similar to the case $X_{0}=$ $\mathbb{P}^{2} \times \mathbb{P}^{1}$ above. The readers can easily redo all the (analogs of) computations and constructions in the previous section.

4.3. The case $X_{0}=$ a complete intersection in $\mathbb{P}^{N}$. Let $X_{0}$ be a smooth projective threefold which is a complete intersection in $\mathbb{P}^{N}$. This means that $X_{0}$ is the intersection of smooth hypersurfaces $D_{1}, \ldots, D_{N-3}$ of $\mathbb{P}^{N}$. By Lefschetz's hyperplane theorem, $X_{0}$ has Picard number 1 . We now show that $X_{0}$ satisfies the conditions of Theorem 5

Lemma 7. Let $\zeta$ be a non-zero movable class in $X_{0}$. Then $\zeta . c_{2}\left(X_{0}\right)>0$.

Proof. Let $d_{1}, \ldots, d_{N-3}$ be the degrees of $V_{1}, \ldots, V_{N-3}$. Let $h$ be the class of a hyperplane on $X$. The Chern classes of the normal bundle $N_{X_{0} / \mathbb{P}^{n}}$ is given by the formula

$$
c\left(N_{X_{0} / \mathbb{P}^{n}}\right)=\prod_{j=1}^{N-3}\left(1+d_{j} h\right) .
$$

In particular,

$$
\begin{aligned}
c_{1}\left(N_{X_{0} / \mathbb{P}^{n}}\right) & =\left(\sum_{j} d_{j}\right) h, \\
c_{2}\left(N_{X_{0} / \mathbb{P}^{n}}\right) & =\left(\sum_{i<j} d_{i} d_{j}\right) h^{2} .
\end{aligned}
$$

From the exact sequence

$$
\left.0 \rightarrow T_{X_{0}} \rightarrow T_{\mathbb{P}^{4}}\right|_{X_{0}} \rightarrow N_{X_{0} / \mathbb{P}^{3}} \rightarrow 0,
$$

and the splitting principle for Chern classes, it follows that

$$
\begin{aligned}
c_{1}\left(X_{0}\right) & =\left.c_{1}\left(\mathbb{P}^{n}\right)\right|_{X_{0}}-c_{1}\left(N_{X_{0} / \mathbb{P}^{n}}\right)=\left((n+1)-\sum_{j} d_{j}\right) h, \\
c_{2}\left(X_{0}\right) & =\left.c_{2}\left(\mathbb{P}^{n}\right)\right|_{X_{0}}-c_{2}\left(N_{X_{0} / \mathbb{P}^{n}}\right)-c_{1}\left(X_{0}\right) c_{1}\left(N_{X_{0} / \mathbb{P}^{n}}\right) \\
& =\left(\frac{(n+1) n}{2}-\sum_{i<j} d_{i} d_{j}-(n+1) \sum_{j} d_{j}+\left(\sum_{j} d_{j}\right)^{2}\right) h^{2} .
\end{aligned}
$$


We have

$$
\begin{aligned}
& \frac{(n+1) n}{2}-\sum_{i<j} d_{i} d_{j}-(n+1) \sum_{j} d_{j}+\left(\sum_{j} d_{j}\right)^{2} \\
= & {\left[\frac{n-4}{2(n-3)}\left(\sum_{j} d_{j}\right)^{2}-\sum_{i<j} d_{i} d_{j}\right]+\left[\frac{n(n+1)}{2}+\frac{n-2}{2(n-3)}\left(\sum_{j} d_{j}\right)^{2}-(n+1) \sum_{j} d_{j}\right] . }
\end{aligned}
$$

By Cauchy-Schwarz inequality, the first bracket on the right hand side of the above expression is non-negative. We now show that the second bracket is positive. We define $x=\sum_{j} d_{j}$. Then $x$ is a positive integer which is $\geq n-3$, and the second bracket is quadratic in $x$ :

$\frac{n(n+1)}{2}+\frac{n-2}{2(n-3)}\left(\sum_{j} d_{j}\right)^{2}-(n+1) \sum_{j} d_{j}=\frac{n(n+1)}{2}-(n+1) x+\frac{(n-2)}{2(n-3)} x^{2}=: g(x)$.

The critical point of $g$ is $x_{0}=(n+1)(n-3) /(n-2)<n$. Hence, to show that $g(x)>0$ for all positive integer $x \geq n-3$, it suffices to show that $g(n-3), g(n-$ $2), g(n-1), g(n)>0$ for any positive integer $n \geq 4$. We now check this latter claim.

For $x=n-3$

$$
g(n-3)=6>0 .
$$

(Note that in this case all $d_{j}$ are 1 and $X_{0}$ is no other than $\mathbb{P}^{3}$.)

For $x=n-2$, using that $(n-2)^{2}>(n-1)(n-3)$, we obtain

$$
g(n-2)>\frac{n(n+1)}{2}-\left(n^{2}-n-2\right)+\frac{(n-2)(n-1)}{2}=3>0 .
$$

For $x=n-1$, we have

$$
g(n-1)=\frac{2(n-2)}{(n-3)}>0 .
$$

For $x=n$, we have

$$
g(n)=\frac{1}{(n-3)}>0 .
$$

A movable class is in particular psef, i.e. can be represented by a positive closed current. Hence, if $\zeta$ is a non-zero movable class on $X_{0}$ then $\xi . c_{2}\left(X_{0}\right)>0$. Hence, Theorem 5 can be applied for such a $X_{0}$.

4.4. A generalization of Theorem [3. The proof of Theorem 6 shows that the conclusion is still valid in the following more general setting. Let $\pi_{1}: X_{1} \rightarrow X_{0}=\mathbb{P}^{3}$ be the blowup at $n$ points $p_{1}, \ldots, p_{n}$. Let $E_{1}, \ldots, E_{n}$ be the exceptional divisors. Let $D_{1}, \ldots, D_{m} \subset X_{1}$ be pairwise disjoint smooth curves. Let $X=X_{2}$ be the blowup of $X_{1}$ at $D_{1}, \ldots, D_{m}$. We define

$$
\gamma:=\sum_{j} \operatorname{deg}\left(\pi_{1}\right)_{*}\left(D_{j}\right) .
$$

Assume that there is $\lambda>0$ such that for any $l$ :

$$
\sum_{j} E_{l} . D_{j} \leq \lambda,
$$


and moreover

$$
\frac{6+\gamma}{\lambda}>\frac{11}{2}
$$

Moreover, assume that for any $j$

$$
\left(\frac{1}{2}+\frac{1}{\lambda}\right) c_{1}\left(X_{1}\right) \cdot D_{j} \geq \frac{g_{j}-1}{2},
$$

where $g_{j}$ is the genus of $D_{j}$.

\section{A possible application to the Ueno's threefold}

Let $E_{\sqrt{-1}}$ be an elliptic curve with an automorphism of order 4 , which we denote by $\sqrt{-1}$. In [41, Ueno asked whether the quotient variety $E_{\sqrt{-1}}^{3} / \sqrt{-1}$ is rational. Campana 9] showed that the variety is rationally connected. Then, by a combination of the two papers [13] and [12, it follows that $E_{\text {sqrt }-1}^{3} / \sqrt{-1}$ is rational. Previously, a similar construction, using instead an elliptic curve with an automorphism of order 3 , has been shown to be rational (see [36]).

The automorphism $\sqrt{-1}$ on $E_{\sqrt{-1}}^{3}$ has 8 fixed points and $64-8$ points of period 2. Therefore, $E_{\sqrt{-1}}^{3} / \sqrt{-1}$ has $8+28=36$ singular points. Let $X_{4}$ be the minimal resolution of $E-\sqrt{-1}^{3} / \sqrt{-1}$, that is $X_{4}$ is the blowup of $E_{\sqrt{-1}}^{3} / \sqrt{-1}$ at the 36 singular points.

Since $X_{4}$ is birational equivalent to $\mathbb{P}^{3}$, by the weak factorization theorem, $X_{4}$ can be obtained from $\mathbb{P}^{3}$ by a combination of smooth blowups and blowdowns. It is then natural to ask the following question:

Question 2. Can $X_{4}$ be obtained from $\mathbb{P}^{3}$ or $\mathbb{P}^{2} \times \mathbb{P}^{1}$ or $\mathbb{P}^{1} \times \mathbb{P}^{1} \times \mathbb{P}^{1}$ by a finite composition of smooth blowups only?

This question is interesting in several aspects. First, the two dimensional analogue, that is the minimal resolution of $E_{\sqrt{-1}}^{2} / \sqrt{-1}$, has been shown to be a finite composition of point blowups starting from $\mathbb{P}^{1} \times \mathbb{P}^{1}$ in [9]. Last, the final proof that $X_{4}$ is rational in [12] is rather abstract. Hence, if the answer to Question 2 is affirmative, it will give an explicit proof that $X_{4}$ is rational.

We note that the smooth threefold $X_{4}$ has automorphisms $f$ coming from the complex torus $E_{\sqrt{-1}}^{3}$ with $\lambda_{1}(f) \neq \lambda_{2}(f)$. Therefore, from the discussion in the introduction of this paper, it is plausible to conclude that the answer to Question 2 is negative. The purpose of this section is to give more weight to this speculation.

We first show that if the answer for Question 2 is affirmative, then centers of the individual blowups must be smooth rational curves. In the below, for any quasiprojective variety $Z$ we will denote by $\chi(Z)$ the Euler characteristic with compact support. For a smooth projective manifold $Z$, we denote by $\rho(Z)$ the Picard number of $Z$.

Theorem 10. Let $X_{0}$ be any smooth projective threefold such that $\chi\left(X_{0}\right)=2+$ $2 \rho\left(X_{0}\right)$ (for example, $X_{0}$ is $\mathbb{P}^{3}, \mathbb{P}^{2} \times \mathbb{P}^{1}$ or $\mathbb{P}^{1} \times \mathbb{P}^{1} \times \mathbb{P}^{1}$ ). Assume that $X_{4}$ can be obtained from the $X_{0}$ by a finite composition of smooth blowups. Then the curves which are centers of the blowups must be smooth rational curves.

Proof. We divide the proofs into several steps. 
Step 1. We claim that $\rho\left(X_{4}\right)=45$. In fact, $E_{\sqrt{-1}}^{3}$ has Picard number 9. Also, the blowup $X_{4} \rightarrow E_{\sqrt{-1}}^{3} / \sqrt{-1}$ has 36 exceptional divisors, one for each singular points. Hence the Picard number of $X_{4}$ is $9+36=45$.

Step 2. We claim that $\chi\left(X_{4}\right)=92$. In fact, first we consider the quotient map $\sigma: E_{\sqrt{-1}}^{3} \rightarrow E_{\sqrt{-1}}^{3} / \sqrt{-1}$. Let $A \subset E_{\sqrt{-1}}^{3}$ be the set of fixed points of $\sqrt{-1}$, and $B \subset E_{\sqrt{-1}}^{3}$ the set of points of period 2 of $\sqrt{-1}$. As mentioned before, the cardinals of $|A|$ and $|B|$ are 8 and 56 , and the cardinals of $\sigma(A)$ and $\sigma(B)$ are 8 and 28. Since the map $\pi: E_{\sqrt{-1}}^{3}-(A \cup B) \rightarrow E_{\sqrt{-1}}^{3} / \sqrt{-1}-(\sigma(A) \cup \sigma(B))$ is a $4: 1$ map, by the excision property we get

$$
\begin{aligned}
\chi\left(E_{\sqrt{-1}}^{3} / \sqrt{-1}-(\sigma(A) \cup \sigma(B))\right) & =\chi\left(E_{\sqrt{-1}}^{3}-(A \cup B)\right) / 4 \\
& =\left(\chi\left(E_{\sqrt{-1}}^{3}\right)-\chi(A \cup B)\right) / 4 \\
& =(0-64) / 4=-16 .
\end{aligned}
$$

Hence, by the excision property, we have $\chi\left(E_{\sqrt{-1}}^{3} / \sqrt{-1}\right)=-16+36=20$.

Next, we consider the blowup $\pi: X_{4} \rightarrow E_{\sqrt{-1}}^{3} / \sqrt{-1}$. This map has 36 exceptional divisors, each is a $\mathbb{P}^{2}$. Since $\chi\left(\mathbb{P}^{2}\right)=3$ and the blowup map is $1: 1$ outside exceptional divisors, arguing as above we obtain

$$
\chi\left(X_{4}\right)=\left(\chi\left(E_{\sqrt{-1}}^{3} / \sqrt{-1}\right)-36 \chi(p t)\right)+36 \times \chi\left(\mathbb{P}^{2}\right)=20-36+36 \times 3=92 .
$$

Here $p t$ denotes a point.

Step 3: $\chi\left(X_{4}\right)=2+2 \rho\left(X_{4}\right)$. This follows from Steps 1 and 2 .

Step 3. Let $Z$ be a smooth projective threefold and $\pi: Z_{1} \rightarrow Z$ a point blowup. Then $\chi\left(Z_{1}\right)=\chi(Z)+2$. This follows easily from the properties of the blowup of a threefold.

Step 4. Let $Z$ be a smooth projective threefold and $\pi: Z_{1} \rightarrow Z$ a blowup at a smooth curve $C \subset Z$. Then $\chi\left(Z_{1}\right) \leq \chi(Z)+2$, with equality if and only if $C$ is a smooth rational curve. Again, this follows easily from the properties of the blowup of a threefold, and the fact that if $C$ is not a smooth rational curve then $h^{1}(C)>0$.

Step 5: final step. If $X_{4}$ is a finite composition of smooth blowups of $X_{0}$, then the number of blowups needed is $\rho\left(X_{4}\right)-\rho\left(X_{0}\right)=45-\rho\left(X_{0}\right)$. From the previous steps we have

$$
92=\chi\left(X_{4}\right) \leq \chi\left(X_{0}\right)+2\left(45-\rho\left(X_{0}\right)\right)=2+2 \rho\left(X_{0}\right)+90-2 \rho\left(X_{0}\right)=92 .
$$

Since equality occurs, it follows from Steps 3 and 4 that the centers of the individual blowups must be either a point or a smooth rational curve.

Now we show how Theorem 10 and Theorems 6,7 and 8 almost give the proof that the answer to Question 2 is negative. In fact, let $X_{0}$ be $\mathbb{P}^{3}, \mathbb{P}^{2} \times \mathbb{P}^{1}$ or $\mathbb{P}^{1} \times \mathbb{P}^{1} \times \mathbb{P}^{1}$. Then, $X_{0}$ satisfies Condition B, while $X_{4}$ does not satisfy Condition B. Assume that $X_{4}$ is a finite composition of smooth blowups starting from $X_{0}$. Let $\pi_{j}: Z_{j+1} \rightarrow Z_{j}$ be an individual blowup in the sequence, where $Z_{j}$ satisfies Condition B. If $\pi_{j}$ is a point blowup then by Theorem 6, $Z_{j+1}$ also satisfies Condition B. If $\pi_{j}$ is the blowup of a smooth curve $C \subset Z_{j}$, then by Theorem 10 we have that $C$ must be a smooth rational curve. If $c_{1}\left(Z_{j}\right) . C \neq 2 g-2=-2$, then by Theorem 8 we have that $Z_{j+1}$ also satisfies Condition B. The remaining case is when $c_{1}\left(Z_{j}\right) \cdot C=-2$. But in this case, half of the conditions of part 2 of Theorem 7 is satisfied. The only condition that is missing is the condition that $C$ is not the only effective curve in its cohomology class. Using part 1 of Theorem 6 , we can also 
show that if the normal vector bundle $N_{C / Z_{j}}$ is not isomorphic to $\mathcal{O}(-2) \oplus \mathcal{O}(-2)$, then $Z_{j+1}$ also satisfies Condition B.

\section{REFERENCES}

[1] D. Abramovich, K. Karu, K. Matsuki and J. Wlodarczyk, Torification and factorization of birational maps, Journal of AMS 15 (3), 2002, 531-572.

[2] T. Bayraktar and S. Cantat, Constraints on automorphism groups of higher dimensional manifolds, J. Math. Anal. Appl. 2013, vol 405, pp 209-213.

[3] E. Bedford, The dynamical degrees of a mapping, arXiv: 1110.1741.

[4] E. Bedford and K.-H. Kim, Dynamics os Pseudo-automorphisms of 3-space: periodicity vs positive entropy, Publ. Mat. 58 (2014), no 1, 65-119.

[5] E. Bedford and K.-H. Kim, Dynamics of rational surface automorphisms: rotation domains, American J. of Math 134 (2012), no 2, 379-405.

[6] E. Bedford and K.-H. Kim, Continuous families of rational surface automorphisms with positive entropy, Mathematische Annalen 348 (2010), no. 3, 667-688.

[7] E. Bedford and K.-H. Kim, Dynamics of rational surface automorphisms : linear fractional recurrences, J. Geom. Anal. 19 (2009), no. 3, 553-583.

[8] J. Blanc, Dynamical degrees of (pseudo)-automorphisms fixing cubic hypersurfaces, Indiana University Math. J. 62 (2013), no 4, 1143-1164.

[9] F. Campana, Remarks on an example of K. Ueno, Series of congress reports, Classification of algebraic varieties, Editors: C. Faber, G. van der Geer, and E. Looijenga, European Mathematical Society, 2011, 115-121.

[10] S. Cantat, Dynamique des automorphismes des surfaces K3, Acta Math. 187:1 (2001), 1-57.

[11] S. Cantat and I. Dolgachev, Rational surfaces with a large group of automorphisms, Journal of the AMS 25 (2012), no 3, 863-905.

[12] J.-L. Colliot-Thélène, Rationalité d'un fibré en coniques, arXiv:1310.5402 Accepted in Manuscripta Math.

[13] F. Catanese, K. Oguiso and T. T. Truong, Unirationality of Ueno-Campana's threefold, arXiv:1310.3569 Accepted in Manuscripta Math.

[14] J.-P. Demailly, Complex analytic and differential geometry, Online book, version of Thursday 10 September 2009.

[15] J.-P. Demailly and M. Paun, Numerical characterization of the Kähler cone of a compact Kähler manifold, Ann. of Math. (2) 159 (2004), no. 3, 1247-1274.

[16] J. Déserti and J. Grivaux, Automorphisms of rational surfaces with positive entropy, Indiana University Math. J. 60 (2011), no 5, 1589-1622.

[17] J. Diller, Cremona transformations, surface automorphisms, and plane cubics. With an appendix by Igor Dolgachev. Michigan Math. J. 60 (2011), no. 2, 409-440.

[18] T-C. Dinh, Tits alternative for automorphism groups of compact Kähler manifolds, Acta Math. Vietnam. 37 (2012), no 4, 513-529.

[19] T-C. Dinh and V.-A. Nguyen , Comparison of dynamical degrees for semi-conjugate meromorphic maps, Comment. Math. Helv. 86 (2011), no 4, 817-840.

[20] T-C. Dinh, V.-A. Nguyen and T. T. Truong, On the dynamical degrees of meromorphic maps preserving a fibrartion, Commun. Contempt. Math. 14 (2012), no 6, 1250042, 18 pp.

[21] T-C. Dinh and N. Sibony, Une borne supérieure pour l'entropie topologique d'une application rationnelle, Ann. of Math. (2) 161 (2005), no. 3, 1637-1644.

[22] T-C. Dinh and N. Sibony, Regularization of currents and entropy, Ann. Sci. École Norm. Sup. (4) 37 (2004), no. 6, 959-971.

[23] T-C. Dinh and N. Sibony, Groupes commutatifs d'automorphismes d'une variété kählérienne compacte, Duke Math. J. 123 (2004), no. 2, 311-328.

[24] M. Gromov, On the entropy of holomorphic maps, Enseignement Math. 49 (2003), 217-235. Manuscript (1977).

[25] P. Griffiths and J. Harris, Principles of algebraic geometry, 1978, John Wiley and Sons, Inc.

[26] R. Hartshorne, Algebraic geometry, Graduate Texts in Mathematics, No. 52. Springer-Verlag, New York-Heidelberg, 1977.

[27] C. McMullen, Dynamics with small entropy on projective K3 surfaces, preprint.

[28] C. McMullen, K3 surfaces, entropy and glue, J. Reine Angew. Math. 658 (2011), 1-25. 
[29] C. McMullen, Dynamics on blowups of the projective plane, Publ. Math. Inst. Hautes tudes Sci. No. 105 (2007), 49-89.

[30] C. McMullen, Dynamics on K3 surfaces: Salem numbers and Siegel disks, J. Reine Angew. Math. 545 (2002), 201-233.

[31] K. Oguiso and F. Perroni, Automorphisms of rational manifolds of positive entropy with Siegel disks , Atti Accad. Naz. Lincei Cl. Sci. Fis. Mat. Natur. Rend. Lincei (9) Mat. Appl. 22 (2011), no 4, 487-504.

[32] K. Oguiso, Free automorphisms of positive entropy on smooth Kähler surfaces, arXiv:1202.2637

[33] K. Oguiso, The third smallest Salem number in automorphisms of K3 surfaces, Algebraic geometry in East Asia, Seoul 2008, 331-360, Adv. Stud. Pure Math., 60, Math. Soc. Japan, Tokyo, 2010.

[34] K. Oguiso, A remark on dynamical degrees of automorphisms of hyperkähler manifolds, Manuscripta Math. 130 (2009), no. 1, 101-111.

[35] K. Oguiso, Automorphisms of hyperkähler manifolds in the view of topological entropy, Algebraic geometry, 173-185, Contemp. Math., 422, Amer. Math. Soc., Providence, RI, 2007.

[36] K. Oguiso and T. T. Truong, Explicit examples of rational and Calabi-Yau threefolds with primitive automorphisms of positive entropy, arXiv:1306.1590

[37] F. Perroni and D.-Q. Zhang, Pseudo-automorphisms of positive entropy on the blowups of products of projective spaces, Math. Ann. 359 (2014), no 1-2, 189-209.

[38] P. Reschke, Salem numbers and automorphisms of complex surfaces, Math Research Letters 19 (2012), no 2, 475-482.

[39] T. T. Truong, On automorphisms of blowups of projective manifolds, arXiv:1301.4957.

[40] T. T. Truong, On automorphisms of blowups of $\mathbb{P}^{3}$, arXiv:1202.4224

[41] K. Ueno, Classification theory of algebraic varieties and compact complex spaces. Notes written in collaboration with P. Cherenack, Lecture Notes in Mathematics, 439 SpringerVerlag, Berlin-New York, 1975.

[42] Y. Yomdin, Volume growth and entropy, Israel J. Math., 57 (1987), 285-300.

[43] D.-Q. Zhang, Automorphism groups of positive entropy on minimal projective varieties, Adv. Math. 225 (2010), no. 5, 2332-2340.

[44] D.-Q. Zhang, The g-periodic subvarieties for an automorphism $g$ of positive entropy on a compact Kähler manifold, Adv. Math. 223 (2010), no. 2, 405-415.

[45] D.-Q. Zhang, Dynamics of automorphisms on projective complex manifolds, J. Differential Geom. 82 (2009), no. 3, 691-722.

[46] D.-Q. Zhang, Automorphism groups and anti-pluricanonical curves, Math. Res. Lett. 15 (2008), no. 1, 163-183.

School of Mathematics, Korea Institute for Advanced Study, Seoul 130-722, RepubLIC OF KOREA

E-mail address: truong@kias.re.kr 